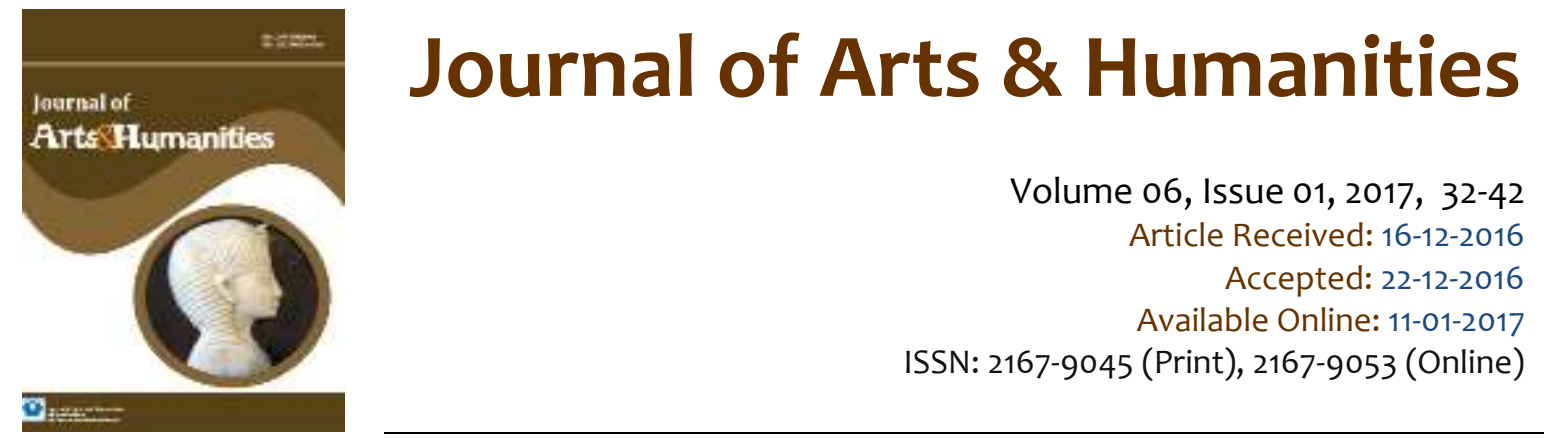

\title{
Balance Toward Language Mastery
}

\author{
Virginia Heslinga, Ed.D. ${ }^{1}$
}

\begin{abstract}
Problems in attaining language mastery with students from diverse language backgrounds and levels of ability confront educators around the world. Experiments, research, and experience see positive effects of adding sign language in communication methods to pre-school and K-12 education. Augmentative, alternative, interactive, accommodating, and enriching strategies using sign language aid learners in balancing the skills needed to mastery of one language or multiple languages. Theories of learning that embrace play, drama, motion, repetition, socializing, and selfefficacy connect to the options for using sign language with learners in inclusive and mainstream classes. The methodical use of sign language by this researcher-educator over two and a half decades showed signing does build thinking skills, add enjoyment, stimulate communication, expand comprehension, increase vocabulary acquisition, encourage collaboration, and helps build appreciation for cultural diversity.
\end{abstract}

Keywords: Accommodations, Autonomy, Diversity, Inclusion, Language, Neuroscience, Signing, Scaffolding. This is an open access article under Creative Commons Attribution 4.0 License.

\subsection{Balance toward language mastery with sign language}

Most people have seen hand motions used in sports to guide players in different functions, often beneath or in spite of the attention of those who are not intended to receive the message. Torres (2013) described the complex sequences sports players learn as they progress to mastery of their chosen sport. Hand signing speeds vary, but depending on the intent and players' knowledge, the signals let a player's central nervous system have some kinesthetic information on necessary changes and perceptions.

People had used hand signs before speech, when they cannot speak, when they had to keep silent, and to supplement speech. Piai, Roelofs, Rommers, and Maris (2015), showed with human brain mapping, that motor aspects with word production can increase memory and understanding of components in spoken communications. Whether one chooses to examine communication abilities psycholinguistically or through motor-control, language via signs with hands, face, and body will appear.

${ }^{1}$ Anna Maria College, Paxton, Massachusetts, United States, Email: vheslinga@annamaria.edu. 
Augmentative and alternative communication (AAC) used to aid children with severe speech challenges and general sign language recognition (SLR) have advanced to provide helpful accommodations and communication options. The purpose of this paper is to show the use of sign language can build thinking skills, increase vocabulary acquisition, encourage autonomy, build acceptance of diversity, promote collaborative efforts, and engender mastery of languages.

The background of this paper stands on experience of an educator who in the 1980 s knew only beginner level American Sign Language (ASL). This educator received deaf and hard of hearing (DHH) students into a heterogeneous class before inclusion was widely practiced in schools in the United States. In this second decade of the twenty-first century, general and special education teachers work as a team for ensuring best practices meet a broad range of needs in an inclusive classroom. Rivera and Antia (2016) showed advances occur and questions arise in using sign language in classes with DHH students and diversity in other student needs and abilities. For inclusive practices, this educator learned not only new teaching strategies but shared more expressively with classes, encouraged students to learn some ASL, to enunciate clearly, and to communicate with facial expressions as much as with words.

Inclusive classroom environments and approaches to teaching present challenges for the range of educational stakeholders. Educators need to learn and practice new skills and strategies. School administrators need to support inclusive practices and environments. School committees need to accept the costs in extra training, changes in setting, materials, and staff. Policy makers and community members need awareness and understanding of providing quality education to all students. The National Disability Resource and Advocacy Centre (NDRAC, 2017) provided guidelines for inclusive schools and for teachers learning sign language for use with all students, as methods to meet diverse inclusive needs. This paper presents research and experience revealing how signing affects thinking skills, reinforces comprehension, and connects to policy ideas and implications for teaching inclusively.

\subsection{Sign language builds thinking skills}

In four research experiments, Borgna, Convertino, Marschark, Morrison, and Rizzolo (2010) found that adding the language experience of signing enriched learning for hearing students more than for deaf or hard of hearing students (DHH). Sign language, added to content and curriculum, advanced comprehension and the scaffolding processes of metacognition in a mainstream environment. With lectures, readings, and activities, tests showed that hearing students learned more than the DHH students when all the participants also had the presentation with an expert signing interpreter. Scaffolding helped students connect sign language to content with an emphasis on main ideas, vocabulary lists, summaries, how and why questions.

Edyburn (2015) showed visual learning methodologies from expressions to motions can increase and inspire students in cognitive and social efforts.

Reddy (2015) showed students with attention deficit hyperactivity disorders (ADHD) need to fidget and move during a school day; motion can actually improve a student's concentration. Using more motions for communication in a classroom provides a purposeful cognitive option allowing movement. Motions such as signs meet a need to move found in kindergarteners through college students' time in classes. Signing strategies aid development of spelling, sounds, modes of communication, and literacy skills. Emmorey and Petrich (2012) showed fingerspelling motions help DHH students to make associations that build knowledge and comprehension of English. Hearing students can gain the same benefits with fingerspelling and spatial visual symbols (Borgia et al, 2010).

The conclusions of Borgna et al (2010) revealed elaboration on reading and strategies for comprehension, along with signing, actively engaged thinking domains in neural paths between right and left sides of the brain. Hearing students also incorporated the signs in their scaffolded organization of ideas for learning content. Using the visual reinforcement for accommodation of DHH students 
enriched learning for hearing students, but did not elicit the same level of higher performance in the DHH students.

Learning another language, including sign language, engages all levels of thinking as described by Bloom, Englehart, Furst, Hill, and Krathwohl (1956): knowledge, comprehension, application, analysis, synthesis, and evaluation. Educators can consider the theory and activities used in second language acquisition (SLA). Vygotsky's ideas on scaffolding and sociocultural theory should help in the understanding of SLA (Lantolf and Poehner, 2014). In reviewing key elements of SLA, Cohalan (2014) described active learning, autonomy, scaffolded learning, critical thinking, learner collaboration, context, authentic communication, and practice. Educators who choose to incorporate the eight elements described by Cohalan (2014) will help students learning the vocabulary, jargon, and idioms of a second, third, or more languages.

\subsection{Active learning}

With mainstreaming and inclusion, students who are deaf or hard of hearing (DHH) will join hearing peers in inclusive classrooms in the United States (US). However, this is not the main reason for teachers to have motivation for learning and sharing sign language. Sign language, from the actual national sign language, engages students actively. For the past 28 years, this educator has used sign language in classes that included immigrants with different levels of the English language, $\mathrm{DHH}$, and hearing students. Elementary, middle school, senior high, undergraduate, and graduate students have learned basics of ASL connected to specific subject content, classroom procedures, conversation, culture classics, warnings, songs, and safety (Heslinga \& Nevenglosky, 2012).

Hogan (2016) described how children, whether autistic, DHH, challenged in some other physical ways, or hearing and able, move through developmental levels. Non-verbal thinking and communication combine with the intrinsic elements of imitation and play. Signing provides an area of strength engaging visual learning and coordination of motor skills. Recognizing differences in motions, sorting, matching, following steps, forming motions promote learning. Vallotton and Ayoub (2010) showed symbols in gestures helped learners to build communication skills in motions, speech, and comprehension.

In this educator's US experience, no student has objected to the incorporation of signing in a classroom. However, some parents of K-12 students objected and wanted to know why their hearing child had to be exposed to and practice American Sign Language (ASL). Conferences and discussions with the parents about learning and the brain, active learning, SLA, and life skills usually engendered a positive point of view of ASL in mainstream classrooms. Students' enjoyment of using signs to communicate, to practice vocabulary, to review procedures, and to share conversation emerged in diverse interactions (Heslinga \& Nevenglosky, 2012).

Parents, guardians, and administrators saw evidence of the positive aspect of ASL motions and expressive possibilities in learning. Students who had difficulty with linear aspects of learning a language suddenly found a freedom and positive focus in the spatial and active movement of learning sign language. Heterogeneous classes show interest and motivation when realizing communication has modes that include expressive motion, active use of symbolic motions, personal and group application for saying a lot silently. The Mayo Clinic (Hoecker, 2016) confirmed teaching ASL to infants and toddlers built effective communication and bonding experiences. Delk (2016) emphasized the many uses of sign language as effective communication for people from infants to elderly. Delk cited Johnson (2012) as a source for explaining more than two dozen professional and personal uses of sign language.

Cozolino (2013) highlighted the important connections between knowledge in neuroscience and learning. Sign language helps teachers who do try to incorporate what neuroscience has discovered about connections with social-emotional learning and active participation in producing and mastering language. Students used ASL to learn cultural elements like the pledge of Allegiance, the national 
anthem, poems, vocabulary, grammar, facts, famous quotes, stories, and lyrics. Students who had difficulty with spelling could feel the form of the word with fingerspelling and made progress in overcoming spelling confusion. Whether a DHH student was present or not, classes found a new sense of involvement that included English Language Learners (ELLs), DHH, and hearing friends throughout the school years.

Cohalan (2013) showed learners build broader connections in thinking, socializing, and problem-solving as they acquire knowledge and skills with a second language. ASL qualifies as a second language in most states in the US. Students see and feel the difference when practicing with the symbols and structure of ASL. The comparing and contrasting that arises in SLA naturally advances thinking skills; Dean, Hubbell, Pitler, and Stone (2012) identified comparing, contrasting, cues, and non-linguistic representations as effective instructional strategies for developing comprehension. ASL added to a classroom uses the strategies to develop comprehension. The sign language of any nation would have the same effect on students in that nation as ASL has in the US. Cognitive development becomes active, interpretive, social, spatial, and visual when one learns sign language.

Intrinsic motivation grows as students experience more social connections and the pride in knowing a set of active symbols that have meaning. Early childhood, kindergarten, and grade one learn alphabets and words faster and with more fun when their hands show the letters and actions connect to meanings. Words with confusing blends and spelling appear more clearly to students who can see and feel the difference in letters through finger spelling in ASL. Although a mainstream class would not have immersion in sign language, the repetitious use of questions, short statements, polite words, and directions in signing does help students to learn and retain the motions and meanings. With more attention paid to creating lessons for varied abilities and learning needs, the use of sign language in a class will enrich communication and socialization (Heslinga \& Nevenglosky, 2012).

Authentic uses for a language also increase motivation. Some students reported their motivation to learn grew when they had a signed short conversation with a deaf child or adult. One student had noticed a deaf person trying to ask a question in a store, and the student could communicate with the deaf person and with the store employee. This experience, recounted to the other students helped stimulate intrinsic motivation. Even young students realized they could communicate in a way many adults could not. They felt empowered in communicating and in helping another person to get a needed item.

Teachers found that when giving instructions, using signs gave visual reinforcement to what was said. One graduate student, a middle school science teacher, who had taught unit vocabulary and safety guidelines with signs, remarked she had never had such a high percentage of students' eyes on her, paying attention to instructions, as when she used signs related to the unit goals and experiments. Students reported they remembered content and instructions in more detail because of signing. This teacher had created a unit checklist for vocabulary that students could review and practice in spelling and in signing with a classmate. A checklist exists for visual comprehension of sign language in $\mathrm{DHH}$, and the check list works with hearing students of other ability levels too (Simms \& Baker, 2014).

Other motivating situations came as a surprise. Teachers who use signing in the classroom found students liked the quiet mode communication, especially during a time when classmates were working with an intense focus on a writing task, quiz, or test. One could ask for more paper, another pencil or pen, or even for permission to go to the bathroom or the nurse without saying anything out loud. The eye contact with the teacher and responses based on requests and comments in signs gave positive reinforcement to the student's thinking and communication skill. In situations requiring long periods of silence or in noisy locations, like attending a sports event, students found the ability to communicate with signing increased enjoyment and a sense of connection.

This educator encountered all of these positive interactions and motivators with pre-school, elementary school, middle school, high school, college, and graduate school students. 
Students also expressed that using sign language, as a class, made them feel more like a united group. ELL students, DHH students, and students with a variety of other learning challenges found they felt a sense of belonging with the class as they signed together. Often the students so adept with reading, writing, and speaking were amazed to find students who struggled with English language had quick abilities in learning and using ASL. Cates, Gutierrez, Hafer, Barrett, and Corina (2013) showed that understanding the use of the body and motions contributes to understanding semantics but the skill of understanding body locations and expressions has a special place in neurobiology.

In an even more unexpected surprise, this educator found students experienced great delight when their lunchroom was told they must have a silent lunch as a punishment because everyone had been too noisy the previous day. Suddenly, the students who had learned some ASL found they could still talk with one another, just using signs. The next time that principal assigned a silent lunch, students were also told to sit on their hands. That principal's instructions left them smiling, even though they were more completely restricted.

When people feel relaxed and interested, learning can occur more easily. Humor can help people to relax while contemplating a topic or lesson. Mohan Raj (2016) described the effective method of incorporating humor into language acquisition as it can lift learning environments socially, psychologically, and academically. Humor can engender a positive spirit in language acquisition. Students often find the ability to state a simple sentence in signed language, like Sorry I'm late, fun. Some find humor in the motions associated with the shape of words and sentences.

New insight into the matter of place, in story telling or referring to characters repeatedly, appears in the process of learning and using ASL. Students will engage their perception in motions and expressions for negative and affirmative declarative sentences and for kinds of questions (Jay, 2011). In signing one must consider referents in terms of character and placement. To have the story understood easily, one must adhere to the location assigned to a character and to express attributes of characters. The physical motions and expressions that accompany the use of sign language also engender a spirit of fun as a total body involvement with language emerges.

\subsection{Increasing autonomy}

Just as human language and brains evolved and adapted; the use of signs in hand, face, and body language has changed. Cozolino (2012) recounted observations on adaptations and innovations of the brain in creating communication and culture. Consider Cozolino's list of nine influential facts about the brain as insight into what the use of sign language will build as autonomy in learners.

1) "The brain is a social organ.

2) The cerebral hemispheres have differentiated from one another and developed specialized functions and skills.

3) Early learning is powerful.

4) Conscious awareness and unconscious processing occur at different speeds, often simultaneously.

5) The mind, brain, and body are interwoven.

6) The brain has a short attention span and needs repetition and multiple-channel processing for deeper learning to occur.

7) Fear and stress impair learning.

8) Our brains have evolved to pay attention to the behaviors and emotions of other people.

9) Learning is enhanced by emphasizing the big picture, then allowing students to discover the details for themselves."

Signing has seemed to connect to learning from the time humans are babies. The fact that babies can learn and communicate in sign language before they can compose verbal sentences demonstrates value in keeping this practice of using motions with speech. Dayanim and Namy (2015) completed experiments showing how babies and toddlers respond to and learn sign language from humans and 
videos. Nursery schools, pre-schools, parents, and guardians have found young children respond easily and well to sign language. Babies and toddlers learn the signs and use the signs to communicate.

Hilger, Loucks, Quinto-Pozos, and Dye (2015) studied production variability in learners. A student's motor control advances just as thinking skills do with the production of symbolic motions, spatial representations, words, phrases, and sentences in sign language. Hilger et al (2015) found that DHH students did try less variability than hearing students in signing the longer they spent working with ASL.

In Autonomy in Language Learning (Menegale, 2013), one sees how children learn language by listening, watching, and mimicking. Play like activities aid in learning a language especially as a person works on constructing familiarity with vocabulary skills. Strategies, interest, motivation and systems of practice with varied methods, enthusiasm, learner-centered choices for students, authentic, and assignments build learner autonomy and thinking skills. Common questions and the logical responses can be practiced almost as a game back and forth from question to response and the facial expressions students will also need to practice help young people learn how to project and read expressions (Duke, 2009).

In becoming autonomous learners, students need to understand and practice mindfulness. Cowan (2010) established and taught guidelines for incorporating mindfulness, and the procedures required use more than once a day. When students learn and use sign language, they think about the words they sign and they think about the words they see other students signing.

Cowan (2010) also showed that making a mindful environment included considerations for others. By teaching students to ask common questions about basic needs in sign and to follow instructions given in sign, classrooms and the school environment have a quiet order considerate of others. Even something like applauding the presentation of a classmate can be done in sign, silently; no other classrooms busy with some other tasks will face interruptions due to the noise of hearing another class applauding.

Sharing also builds mindfulness. Some teachers have found that having learning centers that allow students to share in building sign language skills gives positive collaboration, practice, involvement, vocabulary, and sentence building skills. Solman, in the PBS Newshour (2016) revealed the more people can share, the more they build kindness and compassion. Nurturing values, like kindness and compassion, connect students to mindfulness and their own power to affect others. Realizing their power, self-efficacy, stands as part of building autonomy. Bandura's (1961) work and research confirmed the need for young people to experience positive interactions with others; sharing would provide positive interactions.

\subsection{Problem solving}

Effective language learning finds both teachers and students engaged in problem-solving. When presented with language tasks or any other tasks, participants use a set of metacognitive skills based on scaffolding, comprehension, and risk taking. Vecchio, in Autonomy in Language Learning (Menegale, 2013) how self-regulation will occur as individuals make connections and try communication. A mechanism used while speaking to a class, that increases involvement, like adding signs for letters, words, or concepts, builds practice in approaching challenges from different perspectives, orally and symbolically via motions. Assessment of learners' skills and comprehension also has varied components as the teacher might see via signs that a student understands a concept even when a learner might use the wrong word choice or pronunciation.

\subsection{Lessening stress}

With signing added to communication efforts in language, formative assessments can occur easily, frequently, and naturally-thus avoiding any additional stress that often accompanies assessments. 
Cenoz and Gorter (2008), describing the linguistic landscape, affirmed that students accrued many benefits from exposure to more languages. According to Cenoz and Gorter (2008), five benefits from having multiple languages in an environment include:

- Authentic interactions increase the potential of subtle accumulation of another language.

- Practical experiences with text content and social interaction build academic and social skills.

- Literacy skills advance with growth in multimodal language experiences.

- Having input from written, auditory and active sharing of information stimulates language competence.

- Students will understand language can have powerful effects when shared in different ways.

With the frequent high stakes testing in US public school systems, in this second decade of the twentyfirst century, giving students the opportunity to see, hear, write, and move with language will build selfefficacy and confidence.

Duke (2009) reported a variety of reasons why students benefit from learning ASL. They can have an active method for communication with $\mathrm{DHH}$ and hearing friends who also know sign language thus expanding friendships and deepening relationships. Though signing seems like fun, a most playful visual language, using ASL develops intellectual growth. In using sign language students experience another way of expressing thoughts and emotions. With the incorporation of ASL, language acquisition broadens with vocabulary and skill in forming and interpreting non-verbal communication.

Enns and Herman (2011) suggested that researchers in different sign languages, American, British, and others, should work together to build a measure of sign language assessment. Though no global sign language exists, some motions are understood around the world, and an assessment checklist for signing systems in language acquisition, for English and other languages would help to measure progress. American Sign Language (ASL) has the status of a foreign language in the US, but a standardized test has not yet appeared for ASL; perhaps this contributes to the ways students relax and find enjoyment in the process of learning ASL.

\subsection{Reinforce understanding with sign language}

Although signing does not have the same grammar as spoken and written language, understanding of language increases because of more comprehension of vocabulary. As students understand their own thinking and combinations of ideas, comprehension will grow.

Menegale (2013) described the advantages to any dramatization of reading material and how it illustrates concepts and details. Signing offers the opportunity for dramatization in part or whole of narratives, exposition, vocabulary, actions, and questions.

Duke (2009) explained the opportunities and expectations for motions show learners variations in communication. Roles of reader fall to the one who watches the signer as that one must read the message sent with hand motions, facial expressions, and body language. Students can express self, perform dramatically, engage in everyday chatter, present speeches, play games, give and take quizzes, practice vocabulary for categories, understand and use past, present, and future. In the United States, there are several systems of signing: Manually Coded English (MCE) and signed English which is almost a word for word signing that follows English grammar.

Fant and Fant (2008) emphasized that neither MCE nor signed English is ASL. ASL is the language most DHH people use when communicating with members of the deaf community. ASL does not follow English grammar rules but can help a teacher to show English grammar in counterpoint to ASL. In the second decade of the twenty-first century, young people look more often at phones, i-pads, and other screen technology, than they look at other people. ASL can help students to value observation, socialization, and evaluation skills, and to develop them. 


\subsection{Connecting}

Connections may be a synonym for scaffolding and Reifman (2014) recommended choosing to make connections a habit in life and learning. Schooling should help students develop habits that will strengthen thinking skills. Montessori (2010) often suggested helping children to visualize as a way to engender connections. Sign language consistently helps students to make visual connections with concepts and nouns, concrete and intangible. The habit of speaking with voice and hands can give students opportunities for careers and communication that only linguistic tracks do not include.

Often vocabulary in ASL does not exactly have words common in spoken language. Students have ongoing practice in problem-solving as they make connections between what the English wording and the option for expression a closely aligned message in ASL. Practice, repetition, authentic usefulness, and active engagement create the connections to help individuals learn more than one language. Since ASL serves a distinct culture, DHH, the connections students make in learning ASL will help advance understanding and consideration for a different culture (Reading \& Padgett, 2011).

\subsection{Comparing}

Dean, Hubbell, Pitler, and Stone (2012) explained the benefits of physical and non-linguistic comparisons in engaging students' perception and analysis visual representations. The research efforts that led to Classroom Instruction that Works (Dean, Hubbell, Pitler, Stone, 2012) revealed a list of class activities teachers should include: "Use graphic organizers; make physical models; generate mental pictures; create illustrations; engage in kinesthetic activities." The use of sign language engages all of these recommended learning strategies while reinforcing and expanding language skills. Social interaction with clearly designed activities also promotes learning (Vygotsky, 1978). Menegale (2013) highlighted the need for helping learners to proactively socialize with others as a way of increasing capacity, positive attitude, and motivation; these three elements build autonomy. In signing, students can discern and compare differences in sign formation, facial expression, and layers of description.

According to Krauss and Boss (2013) a pleasurable learning environment allows the brain to stimulate memory, to focus, to feel safe, and to feel brave enough to try new challenges. Students instinctively compare the high stakes testing environments with the tensions that block cognitive abilities; they prefer and desire classes and activities that affirm positive, social, active, and meaningful efforts. Incorporating sign language into class activities and procedures will add to creating a pleasant, active, positive environment.

\subsection{Summarizing}

Using signs stimulates neural pathways on both sides of the brain. Goldin-Meadow (2004) described many values in using gestures in the learning process. Word recognition grows, reading abilities develop aiding literacy and enjoyment in lifelong learning experiences; instruction scores are higher when pre-K children work with signs, and this increase lasts over time. Signs aid learners not only in bilingual classrooms, multi-lingual classrooms, and inclusion classrooms, but also in classrooms of hearing students because active communication with motion promotes learning in a child's development. Just like analyzing and comparing, summarizing stands as a skill that builds thinking skills. Dean, Hubbell, Pitler, and Stone (2012) completed research that showed summarizing helps students make sense of lessons and experiences. Reviewing in summary form with signs can be a whole class, small group, or individual action and the motions will reinforce the main points.

\subsection{Conclusions}

Results of this researcher-educator's years of incorporating sign language into classes have shown students achieve: 
- Faster recognition skills for letters, words, and concepts

- use of manners and considering the needs and views of others

- strengthening memorization skills by adding signing to materials

- visual reinforcement for instructions

- purposeful opportunities for motion

- creative expression through interpretation

- fun in learning

- access to communication even at a distance or amid a noisy environment

- willingness to adhere to rules for silence as communication can still occur

- building a bond with others who know or are learning ASL

- appreciation for language diversity

Policies encouraging Universal Design for Learning (UDL) to meet diverse needs exist across the United States (Test and Bartholomew, 2011). The National Secondary Transition Technical Assistance Center (NSTTAC) promotes adaptations for curriculum and lessons geared to address cultural, gender, age, and ability differences as part of the UDL (Test and Bartholomew, 2011). The main principles of UDL require lessons providing multiple means of representation, action, expressions, and engagement; adding sign language to classes aligns to each of the UDL principles.

The Association for Supervision and Curriculum Development (ASCD) advocates inclusion with strategies that work with a blend of ideas, work in social situations, interaction, interdisciplinary units, authentic activities, and empowering students to engage in the learning processes. The ASCD has found these strategies led to good teaching: relevant, multicultural, and applicable to varied learning abilities. Methods connected to these strategies help classrooms and schools to work according to a least restrictive environment with multiple layers of supports (ASCD, 2017). Using sign language connects to inclusion strategies and student empowerment.

Studies of language development, learning theories, contributions of neuro-scientists, and decades of experience working in mainstream classrooms, K-12, in the US have shown this educator that incorporating sign language in the classroom enriches thinking, socialization, perception, comprehension, analysis, creativity, and appreciation of diverse languages. Bandura's theories of selfefficacy showed that students enjoy learning and feel more at ease when the study of language has social and authentic appreciation of a culture (Mills, Pajaras, Herron, 2007). Bethune saw that effective help for students came through lessons that had a combination of something for the head, something for the heart, and something for the hands framed in respect for others (McCluskey \& Smith, 1999).

In the twenty-first century, educational stakeholders have seen the need for more than high stakes testing. Best practices identify elements of active learning, authentic tasks, interaction, and collaborative efforts reinforcing respect for others. Using sign language in classrooms can provide a healthy balance in cognition, life skills, social interaction, and language mastery.

Policy implications would lead accreditation organizations, administrators, curriculum designers, educators, students, parents, and communities to find and practice a blend of instructional practices that incorporate signing. The methodical use of sign language by this educator over two and a half decades showed signing does build thinking skills, enjoyment, communication, acclimation for English Language Learners to an interactive supportive environment, vocabulary acquisition, collaboration, and appreciation for cultural diversity.

\section{References}

Association for Supervision and Curriculum Development (ASCD). (2017). Policy Priorities. http://www.ascd.org/publications/newsletters/policy-priorities/archived-issues.aspx

Bandura, A. (1961). Psychotherapy as a learning process. (Kindle Edition). USA: All about Psychology. 
Bloom, B., Englehart, M., Furst, E., Hill, W., \& Krathwohl, D. (1956). Taxonomy of educational objectives: The classification of educational goals. Handbook I: Cognitive domain. New York, Toronto: Longmans, Green.

Borgna, G., Convertino, C., Marschark, Morrison C., \& Rizzolo, K. (July 2010). Enhancing Deaf Students' Learning from Sign Language and Text: Metacognition, Modality, and the Effectiveness of Content Scaffolding. Journal of Deaf Studies and Deaf Education, 16(1), 79-100, doi.10.1093/deaf/eng036

Cates, D., Gutierrez, E., Hafer, S., Barrett, R., \& Corina, D. (Summer 2013). Location, Location, Location. Sign Language Studies, 13 (4), 433-461.

Cenoz, J.,\& D. Gorter. (2008). The linguistic landscape as an additional source of input in second language acquisition. International Review of Applied Linguistics in Language Teaching, 46(3), 267287.

Cohalan, E. (Dec 6, 2013). A Review of the Key Factors in Second Language Acquisition. International Conference on Engaging Pedagogy. (ICEP13).Sligo, Ireland.

Costantino, M.A., \& Bonati, M. (2014). A scoping review of interventions to supplement spoken communication for children with limited speech or language skills. Plos One, 9(3), e90744. Doi:10.1371/journal.pone.0090744.

Cowan, M. (13 March 2010). Tips for Teaching Mindfulness to Kids. Greater Good: The Science of a Meaningful life. University of California, Berkeley. Retrieved from http://greatergood.berkeley.edu /article/item/tips_for_teaching_mindfulness_to_kids

Cozolino, L. (19 March 2012). Nine Things Educators Need to Know About the Brain. Greater Good: The Science of a Meaningful Life. University of California, Berkeley. Retrieved from http://greatergood.berkeley.edu/article/item/nine_things_

Dayanim, S., \& Namy, L. L. (May/June 2015). Infants Learn Baby Signs from Video. Child Development, 86(3), 800-811. DOI:10.1111/cdev.12340.

Dean, C.B., Hubbell, E.R., Pitler, H., \& Stone, B.J. (2012). Classroom instruction that works: Research-based strategies for increasing student achievement. (2nd ed.). EAS: McREL.

Delk, K. (3 Jun 2016). ASL, An Innovative Way to Communicate. http://innovatehz.com/asl-innovativecommunication/

Duke, I. (2009). The everything sign language book. (2nd ed.). Avon, MA: Adams Media.

Edyburn, D. (8 September 2015). Visual Learning Methodologies. Inclusive Schools. Retrieved From http://inclusiveschools.org/visual-learning-methodologies-2/

Emmorey K., and Petrich J. A. (2012). Processing orthographic structure: Association between print and fingerspelling. Journal of Deaf Studies and Deaf Education, 17, 194-201. http://jdsde.oxfordjournals.org/content/17/2/194.full.pdf+html, Doi:10.1093/deafed/enro51

Enns, C. J., and Herman, R. C. (Sum 2011). Adapting the, "Assessing British Sign Language Development Receptive Skills Test," into American Sign Language. Journal of Deaf Studies and Deaf Education, 16(3), 362-374.

Fant, L., \& Fant, B.B. (2008). The American Sign Language phrase book. (3rd ed.). NYC, NY: McGraw-Hill Co. Inc.

Goldin-Meadow, S. (Autumn2004). Gestsure's role in the Learning Process. Theory into Practice. DOI: 10.1207/s15430421tip4304_10.

Heslinga, V., \& Nevenglosky, E. (Spring/Summer 2012). Inclusion, Signing, Socialization, and Language Skills. Electronic Journal for Inclusive Education, 2(9). http://corescholar.libraries.wright.edu/ejie/ vol2/iss $9 / 4$

Hilger, A.I., Loucks, T.M.J., Quinto-Pozos, D., \& Dye, M.W.G. (Jul2015). Second language acquisition across modalities: Production variability in adult L2 learners of American Sign Language. Second Language Research, 31(3), 375-388.DOI: 10.1177/0267658315570648.

Hoecker, J.L. (23 Mar 2016). Infant and toddler health. Mayo Clinic. http://www.mayoclinic.org/healthylifestyle/infant-and-toddler-health/expert-answers/baby-sign-language/faq-20057980

Hogan, K. (2016). Nonverbal Thinking, Communication, Imitation, and Play Skills with Some Things to Remember. TEACCH Autism Program. UNC School of Medicine. Retrieved from http://teacch.com/communication-approaches-2/nonverbal-thinking-

Jay, M. (2011). A student's guide to mastering ASL grammar. Los Angeles, CA: Judea Media, LLC. 
Johnson, G. (24 Sep 2012). 32 Uses and Benefits of American Sign Language (ASL) for Silent Communication. Resources for Life. http://www.resourcesforlife.com/docs/item1560

Krauss, J., \& Boss, S. (2013). Thinking through project-based learning. Thousand Oaks, CA: Sage Publications.

Lantolf, J. P., \& Poehner, M.E. (2014). Sociocultural theory and the pedagogical imperative in L2 education: Vygotskian praxis and the research/practice divide (ESL \& Applied Linguistics Professional Series). NYC, NY: Routledge.

Menegale, M. (Ed.). (2013). Autonomy in language learning: Getting learners actively involved. Canterbury, UK: IATEFL, Darwin College.

Mills, N., Pjares, F., \& Herron, C. (2007). Self-efficacy of college intermediate French students: Relation to achievement and motivation. Language Learning, 57(3), 417-422. doi: 10.1111/j.1467-9922.2007. 00421.x

Montessori, M. (2010). The absorbent mind. Poland: Beta Nu Publishing.

Mohan Raj, S. (2016). Role of Humour in Second Language Teaching and Acquisition. Language in India, 16(4), 27-33.

National Disability Resource \& Advocacy Center (NDRAC, 2017). Inclusive Education. NDRAC. http://ndrac.weebly.com/inclusive-education.html

Piai, V, Roelofs, A., Rommers, J., \& Maris, E. (July, 2015). Beta Oscillations Reflect Memory and Motor Aspects of Spoken Word Production. Human Brain Mapping. 36(7), 2767-2780. MEDLINE Complete, EBSCOhost.

Reading, S., \& Padgett, R. J. (Dec 2011). Communication Connections: Service Learning and American Sign Language. American Journal of Audiology, 20. doi: 10.1044/1059-0889(2011/10-0029).

Reddy, S. (22 June 2015). The Benefits of Fidgeting for Students with ADHD. Wall Street Journal. http://www.wsj.com/articles/the-benefits-of-fidgeting-for-students-with-adhd-1434994365

Reifman, S. (2014). 22 Habits that empower students: How to develop habits of Mind and habits of character in the elementary classroom. USA: Amazon Media.

Rivera, M. C., \& Antia, S. D. (1 Apr 2016). Inclusive Education for Students Who Are Deaf and Hard of Hearing. Raising and Educating Deaf Children. Oxford University Press Journals. http://www.raisingandeducatingdeafchildren.org/2016/04/01/inclusive-education-for-students-whoare-deaf-and-hard-of-hearing/

Simms, L., \& Baker, S. (2014). Visual Communication and Sign Language Checklist for Deaf and Hard of Hearing Children (VCSL). Gallaudet University, Retrieved from http://vl2.gallaudet.edu/resources |vcsl/

Solman, P. (9 June 2016). How do humans gain power? By Sharing it. PBS Newshour. Retrieved from http://www.pbs.org/newshour/bb/how-do-humans-gain-power-by-

Test, A. W., \& Bartholomew, A. (2011). Universal Design for Learning and Secondary Transition Planning for Students with Disabilities: 101. National Secondary Transition Technical Assistance Center. http://ncdcdt.org/wp/wordpress/wp-content/uploads/2011/05/UDL_Transition.pdf

Torres, E.B. (2013). Signatures of movement variability anticipate hand speed according to levels of intent. Behavioral and Brain Functions: BBF, 910. doi:10. 1186/1744-9081-9-10.

Vallotton, C. D., \& Ayoub, C. C. (2010). Symbols build communication and thought: The role of gestures and words in the development of engagement skills and social-emotional concepts during toddlerhood. Social Development, 19(3), 601-626. doi:10.1111/j.1467-9507.2009.00549.x

Vygotsky, L. S. (1978). Mind in society: The development of higher psychological processes. Cambridge, MA: Harvard University Press. 